\title{
Las revistas técnicas como base documental para la recuperación de un patrimonio olvidado: el caso de las salas de cine españolas
}

\author{
Technical journals as a documentary basis for recovering \\ forgotten heritage: case study of spanish cinema theatres
}

\author{
A. Castillo(*), $\underline{\text {. Azorín }}^{(*)}$
}

\section{RESUMEN}

Este trabajo consiste en una valoración de las revistas técnicas españolas como fuentes de información para la recuperación del Patrimonio Cultural. Para mostrarla, se han seleccionado como caso de estudio las salas de cine, que como construcciones singulares se desarrollaron especialmente entre los años 20 y 60 del siglo recién terminado. El método de trabajo para valorar estas obras ha consistido en realizar una revisión bibliográfica y documental de las revistas- así como de otros trabajos vinculados con la Historia de estos inmuebles-, para finalmente seleccionar tres de ellas; Cortijos y Rascacielos, Arquitectura/Revista Nacional de Arquitectura e Informes de la Construcción, puesto que a lo largo de las décadas citadas son las que dedican más páginas a este tipo de arquitectura. A través de un estudio comparativo de estas publicaciones periódicas, hemos podido comprobar la importancia que su uso puede tener para el tratamiento del Patrimonio Cultural, observando cuáles son las características y peculiaridades que más se resaltan sobre las obras realizadas desde el punto de vista constructivo, inclusive, sobre otros aspectos relacionados con las características que definen y diferencian entre ellas a cada una de estas publicaciones periódicas.

146-107

Palabras Clave: análisis documental, revistas técnicas españolas, construcción de salas de cine, Patrimonio Cultural.

\begin{abstract}
SUMMARY
This paper assesses Spanish technical journals as sources of information to recover the nation's cultural heritage. To this end, a case study was conducted of cinema theatres, buildings erected as distinctive structures essentially in the nineteen twenties through the nineteen sixties. The method used to evaluate these buildings consisted in a bibliographic and documentary review of periodicals, as well as other papers relating to the history of these buildings, to ultimately select three journals for study: Cortijos y Rascacielos, Arquitectura/Revista Nacional de Arquitectura and Informes de la Construcción, the three that devoted most space to that type of architecture in the five decades mentioned. A comparative study of these periodicals revealed the importance of their use in connection with the Cultural Heritage, observing the emphasis placed on the characteristics and peculiarities of the works involved from the standpoint of construction, even over other factors relating to the characteristics that define and differentiate these journals.
\end{abstract}

Keywords: documentary analysis, spanish technical journals, cinema theatre construction, Cultural Heritage.

\footnotetext{
(*) Facultad de Geografía e Historia. Universidad Complutense de Madrid (Madrid, España)

${ }^{(* *)}$ Instituto de Ciencias de la Construcción Eduardo Torroja-CSIC (Madrid, España)
} 


\section{INTRODUCCIÓN}

Existe en España multitud de publicaciones a través de las cuales se relaciona el cine con la Arquitectura, con la escenografía e incluso con la construcción de algunas casas de famosos cineastas que se diseñaron tomando como modelo películas concretas. Llama igualmente la atención en estos escritos el diseño y la construcción de las salas de cine, aspecto sobre el que se centra este trabajo.

A pesar de que las salas de cine fueron obras singulares de la arquitectura española, ya que en muchas ocasiones fueron proyectadas por notables arquitectos cuyos encargos eran de promotores que no escatimaban en costes y pugnaban por su magnificencia (1) (De la Madrid 1996b), la especulación voraz de finales del siglo XX, el cambio de usos y costumbres de la sociedad, junto al avance de las nuevas tecnologías en reproducción y visualización de imágenes (por ejemplo el Home Cinema), provocaron la desaparición de muchas de ellas.

Aunque tardíamente con respecto a otros países en los que se dio una situación similar, en los años 80 del S. XX nace un pequeño movimiento en nuestro país a favor de la conservación y la protección de estos edificios, sin embargo esta preocupación social, aunque llevada a la prensa en más de una ocasión, no ha impedido todo lo que debería su destrucción (2).

Prueba de ello es que apenas se han declarado como Bien de Interés Cultural (BIC) edificios diseñados como cinematógrafos. Una ojeada a la bases de datos facilitada por el Ministerio de Cultura a través de su página web (www.mcu.es) nos muestra que entre incoados y declarados no llegan a los 5. Es más, existe un caso que después de haber sido incoado como BIC, su declaración fue desestimada (3).

Las Comunidades Autónomas han llevado a cabo algunas inscripciones o inventarios a través de sus leyes de Patrimonio Histórico o Cultural y sus propias figuras de protección. Pero llama la atención que la mayoría son muy recientes (de después de 2000). Así por ejemplo, en Andalucía se destacan las edificaciones vinculadas al movimiento moderno, mientras que el Inventario de Patrimonio Arquitectónico Catalán incluye 43 cines. Rara vez se utilizan figuras de máxima protección, aunque existen algunos casos, como en Baleares, donde se declaró BIC el Teatro Pereira de Ibiza, utilizado como cine durante todo el S. XX. Por ello se ha creído necesario enfocar esta investigación hacia el apoyo a la recuperación de este Patrimonio Cultural, al entender que el cine, y en consecuencia las salas donde se proyectaba, marcó especialmente el S. XX y es un legado importante de nuestra Historia más reciente.

\section{APROXIMACIÓN A LA HISTORIA DE LAS SALAS DE CINE EN ESPAÑA}

Desde que los hermanos Lumiére dieran a conocer su invento de presentar imágenes en movimiento (1895), no llegó a pasar más de un año para que el cinematógrafo llegase a España. Según la prensa de la época, se considera que la primera emisión cinematográfica madrileña se llevó a cabo en la Carrera de San Jerónimo, en un local dedicado a la fotografía, ubicado en los bajos del Hotel Rusia (4) (Seguin y Letamendi 1996:28-33). Aunque al parecer llegó antes a Barcelona, principalmente por su proximidad a Francia, donde se proyectaron las primeras imágenes cinematográficas en el estudio fotográfico de Antonio y Emilio Fernández "Napoleón", sito en la Rambla de Santa Mónica, n 15-17 (5) (González 1996: 220). A partir de estos dos focos iniciales, las exhibiciones se extendieron rápidamente por todo el territorio nacional.

Los primeros espacios para el cine fueron los locales de los espectáculos ya existentes, incluidos los teatros, teatros-circos, barracas y similares (6) (Pérez Rojas 1985, (7) De la Iglesia et al. 1990 y (8) Carrasco 1992). Era suficiente que dispusiesen de un espacio para el proyector y una superficie donde se pudiera ver la imagen de aquellas primeras películas sin sonido y en blanco y negro. De hecho, podemos considerar al cine de esta primera época como una parte más del espectáculo ferial y de variedades que se exhibía por pueblos y ciudades. Habrá que esperar unos años más, y será después de la I Guerra Mundial, durante los años 20, cuando comiencen a edificarse espacios más específicos pensados y diseñados para el cine (9,10) (De la Madrid 1996a:19 y Fernández 1988:273).

Se seguirán proyectando películas en algunos de los espacios lúdicos existentes, al mismo tiempo que muchos teatros se siguen utilizando para este fin. De hecho, es claro que la adecuación del espacio para el cine nace del propio teatro. Hasta la Guerra Civil española, el desarrollo de este tipo de inmuebles estuvo caracterizado por buscar un protagonismo en la ciudad en lugares céntricos, incluidos dentro de grandes proyectos para edificios residenciales o de oficinas. En cuanto a su interior, las salas van perdiendo poco a poco las tradicionales formas circulares, elípticas, etc., propias del teatro o el music-hall, para volverse rectangulares. La atención del espectador debe centrarse sólo en la pantalla, lo cual 
influirá de modo notable en la distribución y forma de las zonas de asientos. Las butacas se diferencian escasamente y los pisos altos, que en algún caso aún mantienen palcos, se consideran zonas preferentes.

A finales de los años veinte del siglo pasado, se inicia la construcción de grandes salas más elegantes y confortables. Los locales están dotados de amplios vestíbulos, generalmente con bar, destacando su profusa decoración en pinturas y vidrieras. Los estamentos sociales más privilegiados comienzan a tener en el cine una referencia, un modo de diversión, lo que le convierte en espectáculo de interés social como ya lo eran el teatro, la zarzuela o las variedades en sus distintas facetas. En el diseño de las salas se introducen innovaciones técnicas que llegan de la mano de los arquitectos más distinguidos del momento, siendo los proyectos de los edificios de gran complejidad y variedad con diversidad de estilos arquitectónicos en consonancia con el panorama arquitectónico del momento.

Estos cambios que se observan en la arquitectura cinematográfica madrileña se imitarán en el resto de España. En estos momentos, la Arquitectura española estaba sumida en una confusión, recuperando estilos del pasado en ocasiones, y sin un claro desarrollo de la modernidad como ocurría en otras partes de Europa. Las innovaciones técnicas más notables aparecen tanto entre las grandes salas como en las pequeñas (11) (Fernández 1988: 273-278).

En las zonas más céntricas de las ciudades, especialmente en Madrid, los cines no sólo se construyen en edificios exentos de gran representatividad, sino que también se ubican en los bajos de los dedicados a viviendas y oficinas, mientras que en los barrios periféricos emergentes son edificios exentos dedicados al ocio y que albergan la sala de cine, un bar y a veces sala de fiestas.

El apogeo del espectáculo cinematográfico se produce en los años 30, con la edad dorada del cine de Hollywood (12), coincidiendo con la entrada en el mercado cinematográfico del sonido y el color en las películas. Este nuevo sistema se generalizó en España a comienzos de los años 50, llegando a desplazar como opción de ocio a los espectáculos clásicos como eran el teatro, la zarzuela o el music-hall.

En estos años es cuando más atención se presta a la construcción de espacios dedicados a la exhibición de las películas. Se introduce la arquitectura "art-decó" en los cines de la mano de Luís Gutiérrez Soto que, como experiencia vanguardista, unida al racionalismo emergente, dio lugar a una arquitectura moderna muy interesante. En el cine Callao (1926-1927) se deja influir por un art déco que revisará en sus siguientes proyectos del cine Europa (1928) y Cine Barceló (1930), donde depura las líneas expresivamente $y$, al solucionar un edificio en esquina, se acerca a la lección dada por E. Mendelhson y R. Neutra en el Berliner Tageblatt (1921-1923. Berlín).

Desde esta década, hasta la de los 70, muchas revistas técnicas recogen gran cantidad de trabajos sobre cines, españoles y extranjeros, como ejemplos de nuevas arquitecturas. Se abre un periodo para el diseño, en el que la acústica y las grandes dimensiones de las salas toman un papel relevante.

El interés que despierta este tipo de edificios hace que el Ministerio de Instrucción Pública cree un concurso Nacional de Arquitectura sobre cines de más de 200 espectadores en diversas regiones. Su objetivo es la obtención de proyectos orientadores para provincias. Se multiplica el número de salas y también las tipologías, especialmente en los años 40 y 50. Al mismo tiempo que se desarrollaron cines modestos (como los de barrio o los de verano), nacían otros más espectaculares, ubicados en los centros de los pueblos (cines rurales) o en las grandes ciudades.

Las razones para este apogeo son varias. En primer lugar por ser una novedad atractiva para la mayoría de gente, especialmente para una burguesía incipiente que mostró un gran interés por el cine, en detrimento del teatro y las variedades. En segundo lugar, por su coincidencia en el tiempo con la Guerra Civil y la posguerra española, que lo convierte en refugio y entretenimiento ideal.

La aparición de la TV (13) y el video marcan claramente el inicio de una nueva época para la arquitectura del cine que entra en crisis a partir de los 60 llegando al máximo declive en los años 80. Las salas se transforman para intentar ser rentables económicamente. Es la época de los multicines y de los cines en centros comerciales, lo que provoca el cierre de muchas salas y que continúan desapareciendo en la actualidad (14) (Balsalobre García 2006:133). Otra de las razones es el cambio de gusto en la sociedad, que prefiere salir a los centros comerciales, a las afueras de las urbes o a zonas menos céntricas, para ir al cine.

Desde los años ochenta hasta hoy, apenas encontramos cines originales más allá de excepciones vinculadas a la aplicación de nuevas tecnologías, como pueden ser los IMAX o similares, de tres dimensiones. El 
centro de la ciudad se dedica al comercio y la restauración que se ubican, en muchas ocasiones, en los espacios cinematográficos singulares edificados con anterioridad. Esto no significa el fin de las salas de cine, sino un cambio de ubicación más hacia la periferia.

\section{LAS REVISTAS TÉCNICAS Y SUS PUBLICACIONES SOBRE LAS SALAS DE CINE}

Sobre la Historia de las salas de cine en España hay numerosas fuentes de información que permiten hacer un recorrido general, incluso, de una manera detallada para el caso de algunas ciudades, como Madrid, Barcelona o Alicante, así como de Comunidades Autónomas como Andalucía (15) (De la Iglesia Salgado y col. 1990), Asturias (Cinematógrafo y Varietés 1996) o Zaragoza (16) (Biel Ibáñez 1994-95: 297-315), etc. En ellas se aportan desde planos generales de los edificios, fotografías de sus fachadas e interiores, hasta las carteleras de las películas que se proyectaron.

Sin embargo, desde la perspectiva de la Historia de la construcción, en el sentido de los aspectos técnico-constructivos, apenas hay trabajos que aporten información de manera conjunta (17). No obstante, existen gran cantidad de textos que aparecen en las publicaciones periódicas especializadas y que permiten tener una visión más global sobre esta arquitectura.

Por este motivo la intención de este trabajo es la recopilación y análisis de una literatura dispersa y de difícil acceso y que como fuentes de información hasta ahora no han sido valoradas. Nos estamos refiriendo a las principales revistas técnicas españolas por ser las fuentes transmisoras de los avances de los sistemas constructivos científico-técnicos a lo largo de más de 150 años. Además, la regularidad que presenta este tipo de publicaciones las hace ser valedoras como fuentes de investigación histórica.

A través de sus páginas dan a conocer a los técnicos especialistas los edificios más representativos e impactantes tanto nacionales como internacionales, lo cual les permite ser también fuente de primera mano para el estudio de la evolución de la arquitectura e ingeniería, siendo el principal vehículo de intercambio de información de los logros y avances científicos de las principales obras que se llevan a cabo a lo largo de este dilatado periodo. Se ha llevado a cabo una búsqueda selectiva a través de índices de las revistas, repertorios bibliográficos y bases de datos bibliográficas (18). Partiendo de los datos obtenidos se hace un análisis de los resultados. La mayoría de los artículos que aparecen en las revistas coincide con el apogeo de las salas de cine en España, que, como veíamos anteriormente, se da entre los años 20 y los 60 del siglo pasado. Estos trabajo aparecen especialmente en Cortijos y Rascacielos, Arquitectura- denominada durante una época Revista Nacional de Arquitectura (ver más adelante)- e Informes de la Construcción, aunque también se han localizado unas pocas revistas que recogen obras a partir de los 80 e incluso 90 de este siglo (19), resultando más interesantes los trabajos recogidos en publicaciones más clásicas y de la primera etapa.

El contenido general de estos artículos tiene una estructura y tipo de información similar. En la mayoría de las ocasiones se resume la razón de la obra y la problemática que la hizo objeto de su publicación, bien fuera por diseños innovadores, adaptación a distintos espacios o construcciones e incluso renovaciones. Datos como dimensiones, aforos, distribución de espacios, especificándose las salidas de emergencias, iluminación, climatización, etc., son los más comunes.

Obviamente, la referencia al año y al arquitecto que diseñó el edificio también es una constante en estos textos. A veces, se recogen incluso los presupuestos. Generalmente son proyectos a modo de ejemplo de distintos lugares, normalmente en ciudades, aunque en ocasiones también se incluyen trabajos en espacios al aire libre, especialmente en pequeñas localidades. Además del uso de materiales o soluciones arquitectónicas innovadoras, tanto desde el punto de vista estético como decorativo del interior y el exterior de la sala, son reiterativos los temas vinculados a la seguridad, el confort, la ventilación o climatización, la iluminación y el sonido (acústica).

Relativo a estos últimos temas, y en especial en relación con la seguridad y el sonido, hay que comentar que resulta lógica su constante presencia si se tiene en cuenta la legislación vigente durante los años de apogeo de los cines. Sin duda, la seguridad en relación a las instalaciones específicas y a la propagación de fuego son los temas que más preocupan en la normativa. Junto a ellos, existe bastante articulado dedicado a la construcción y reforma, al alumbrado, calefacción y ventilación (20). Ello explicaría la falta de detalle en las publicaciones sobre otros aspectos constructivos que los Reglamentos de Policía de Espectáculos ya recogían. Debemos pensar que al lector al que va dirigida la información no le interesa especialmente este aspecto.

Finalmente, como es común a las publicaciones de este tipo, el grafismo y la imagen tie- 
nen un gran peso. Unas veces se cargan las tintas en los planos generales o de detalles sobre las construcciones, en otras ocasiones es la fotografía la que invade las páginas. Todo ello deja claro que la intención es mostrar el viejo lema de "una imagen vale más que mil palabras" y que estas publicaciones se caracterizan por su pragmatismo, siendo conscientes de que será ese discurso tan directo el que atrape al lector especializado.

A continuación se presenta un resumen descriptivo del contenido de estos artículos en las tres revistas citadas anteriormente y que se han seleccionado en virtud de la importancia que han dado a este tema, entendiendo con ello que su contenido resulta clave para recuperar los viejos cines que aún nos quedan (21).

\section{-Cortijos y Rascacielos. Casas de Campo. Arquitectura. Decoración}

Es una revista bimensual dedicada a la arquitectura y editada en Madrid desde 1930 a 1954 con un paréntesis entre los años 19361943. Fundada y dirigida por el arquitecto Casto Fernández-Shaw es una publicación caracterizada por la simplicidad y claridad en planteamiento, fruto de su mano y pensamiento, como una de las publicaciones que tanto en España como en el extranjero, dieron lugar a capítulos peculiares de la prensa de arquitectura de su época.

Desde 1933 se ha publicado en esta revista información sobre 12 salas de cines. Dos se corresponden a la primera época siendo uno de ellos el cine Actualidades en Madrid, obra de Ulargui ya publicado en Arquitectura (22), mientras que el otro artículo da noticias sobre el fallo del Primer Premio del Concurso Nacional de Arquitectura sobre un proyecto de cine al aire libre y cuyo ganador fue Aníbal Álvarez (23), Figura 1.

Es en la década de los años cuarenta cuando se recogen más noticias sobre salas cinematográficas haciendo incidencia en las de fuera del ámbito madrileño. De este modo se describe un cinematógrafo en Málaga de (24) González Edo, el Cine La Coruña de (25) López Delgado, el Cine Montecarlo de Ricart Biot ${ }^{26}$, el cinematógrafo de Borja (Zaragoza) de (27) Santiago Laguna Mayandía, así como el cine Avenida de San Luís de Potosí de (28) Eduardo Robles.

Referente a los cines madrileños se recogen cuatro salas que se publicaron entre los años 1945 y 1947 (29).

En general, estos trabajos aportan más información gráfica que textual y las descripciones de los edificios son muy someras

haciendo referencias a los temas ya destacados para el conjunto de estas revistas.

\section{-Arquitectura/Revista Nacional de Arqui- tectura}

En 1918 La Sociedad Central de Arquitectos crea como órgano de representación profesional Arquitectura, Revista mensual ilustrada, Órgano Oficial de la Sociedad Central de Arquitectos. En 1931 se constituye el Colegio de Arquitectos de Madrid cuya Junta decide hacerse cargo de la edición de la revista Arquitectura a partir de enero de 1932 hasta 1936. Durante la posguerra y más concretamente, a partir de 1941 se reinicia bajo el amparo de la Dirección General de Arquitectura con el título de Revista Nacional de Arquitectura y no será hasta 1946 cuando vuelva a editarse en el Colegio Oficial de Arquitectos de Madrid hasta 1958. Desde 1959 hasta la actualidad continúa editándose con el nombre de Arquitectura. No está considerada como una revista totalmente técnica, sino arquitectónica, aunque sí es referente en la divulgación de las innovaciones y tendencias por las que ha pasado la arqui-

1. Proyecto de cine al aire libre. Concurso Nacional de Arquitectura. (Fuente: Cortijos y Rascacielos, $\mathrm{n}^{\circ}$ 16, 1934: 21).
21

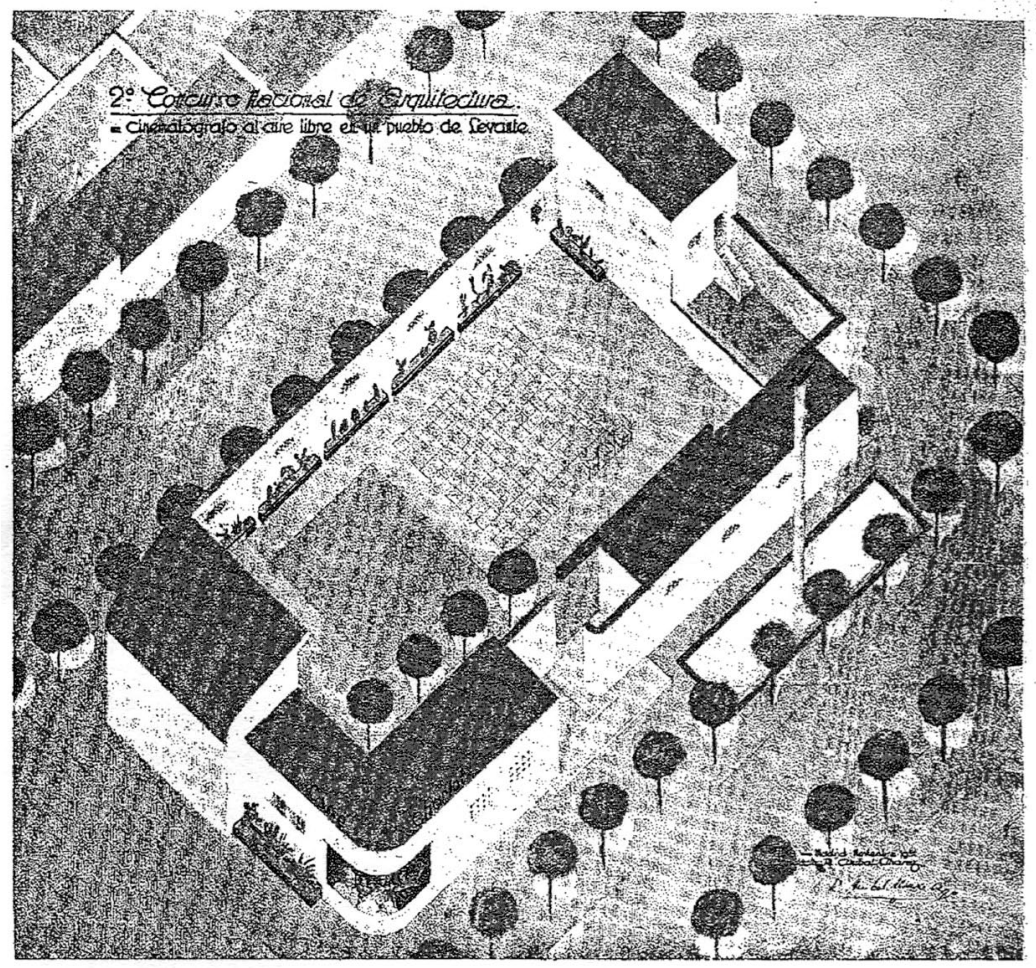

Concurso Nacional de Ãrquitectura

\section{Proyecto de cine al aire libre (Clima cálido seco)}

Arquitecto: Aníbal Alvarez. 
tectura española moderna, especialmente la madrileña.

En la búsqueda realizada se han encontrado, a partir de 1926, un total de 35 trabajos que han sido agrupados por décadas.

En los años 20, aparecen reseñadas en artículos diferentes, con más o menos entidad, las siguientes salas cinematográficas españolas, todas ellas ubicadas en Madrid.: El Palacio de la Música (30) (Zuazo, 1926), el cine Callao (31) (Gutiérrez Soto, 1927) y el cine San Carlos (32) (Lozano Lardet, 1929).

De ellas, destaca el trabajo de Luis Gutiérrez Soto dedicado a explicar su proyecto sobre el cine Callao de la Gran Vía madrileña. El autor comienza justificando su obra adaptada a la peculiar forma del solar. Describe desde detalles constructivos, materiales empleados, hasta la orientación artística que ha seguido en la ejecución de la obra. A lo largo de las 15 páginas que tiene el artículo se puede conocer la solución dada a los accesos al edificio, la decoración -especialmente la pintura-, la iluminación y la acústica y a los costes de la obra. El autor también valora el estilo elegido para la fachada. El texto se complementa con numerosos planos y fotografías.

En esta década también aparecen dos reseñas de salas extranjeras, concretamente de Berlín, cuya obra fueron realizadas por Poelzig (33) (García Mercadal) y (34) Mendelhson. Las descripciones son muy someras complementándose con fotografías tanto del interior como del exterior de los edificios. Se hace también una breve referencia a la forma, el aforo, la acústica, la iluminación y la decoración.

En la década siguiente (años 30) aparecen siete artículos dedicados a las salas cinematográficas. Es un periodo en el que hacen furor los "concursos", y es así como en 1931 se convoca un concurso "privado" para la edificación en un inmueble en la Plaza del Callao de Madrid y cuyo promotor fue el marqués de Melín. El concurso fue anulado y el proyecto se encomendó finalmente a los arquitectos Feduchi y Eced para la realización del edificio Carrión.

Como se ha adelantado en el apartado histórico, también en 1931 se convoca por parte del Ministerio de Instrucción Pública un Concurso Nacional de Arquitectura con objeto de obtener proyectos que puedan ser orientadores para la realización de cinematógrafos en diversas regiones de España y cuyo aforo no superase las 200 butacas. El fallo de este concurso se dio a conocer en Arquitectura en 1932 (35). En este mismo año se reseñan los cines Fígaro (36) (F. López Delgado:1932) y Tetuán (37) (Riancho y Torriente: 1932), ambos madrileños.

El número 1 del volumen XVII de la revista correspondiente a los años 1935-36 está dedicado al citado edificio Carrión de (38) Luis Martínez Feduchi y Vicente Eced y Eced, que incluye una sala de cine. El trabajo consta de 32 páginas en las cuales se describe ampliamente el proyecto que fue ganador del primer premio del concurso de anteproyectos de 1931 y de la segunda medalla de la Exposición de Bellas Artes de 1934. Los autores justifican el diseño del edificio en base a la superficie del solar, detallan el proceso constructivo, el tiempo de ejecución de la obra, su estructura -en la que se introduce la famosa viga vierendell- y el estilo de la fachada.

Se recrean en la ordenación de los espacios dedicados a los servicios, los materiales empleados tanto en la construcción como en la decoración interior, con desplegables en los que aparecen detalles del diseño del mobiliario y que complementan el abundante material gráfico compuesto de planos y fotografías, Figuras 2 y 3 .

Además en este año se publica la reforma del cine Carretas (39) (José Sanz y José Fonseca) en la que los arquitectos responsables de la misma manifiestan las dificultades de este proyecto, especialmente referido a la climatización, la ventilación y sobre todo la acústica, dando más importancia a la confortabilidad del local que a la decoración que, sin embargo, no olvidan. Para lograr el confort acústico los autores manifiestan de modo exhaustivo la serie de ensayos Ilevados a cabo con objeto de conseguir este objetivo.

El último cinematógrafo reseñado en Arquitectura durante la década de los 30 es el cine Salamanca (40) (Francisco Alonso Martos, 1936). En las páginas de este artículo se describe de forma somera el edificio concebido como sala de cine clásica de la época en la que se incluye sala de fiestas y como viene siendo generalizado en todos las publicaciones de este tipo, se hace mención a la confortabilidad, iluminación, acústica y decoración acompañada de fotografías y planos. En la década de los 40 no se publicaron trabajos destacables sobre salas de cine. En 1943 se hace una recensión sobre el cine Victoria de Madrid que, proyectado por Gutiérrez Soto en 1934, se finalizó en 1936. Este edificio fue catalogado como cine de barrio o reestreno e incluye la típica sala de fiestas en el sótano. El trabajo tiene poco texto y está acompañado por tres fotografías.

En 1944 aparece un artículo donde se hace referencia a la adaptación de una parte de 
los bajos del Hotel Palace como sala de cine, así como a los problemas que plantea el cambio de uso en la ventilación y la acústica. El proyecto lo llevaron a cabo conjuntamente los arquitectos (41) López Izquierdo y Durán Cottes.

A mediados de los años 40 comienzan a referenciarse en esta revista los cines de provincias y así en 1946 aparece la reforma del cine Novedades de San Sebastián (42) (Aguinaga y Domínguez Salazar), en 1949 la también reforma del Dorado de Zaragoza (43) (Santiago Lagunas) y el proyecto del Aliatar de Granada (44) (Prieto Moreno). Son descripciones someras de las obras acompañadas de material gráfico.

La década de los 50 puede considerarse especial para la revista en cuanto a la información que aporta para el estudio de la arquitectura de las salas de cine en España.

En 1951 se dan noticias sobre el cine Niza de Barcelona (45) (Aixela y Tarrega) entre las que destacan su estructura de hierro laminado; del cine Postas de Madrid (Juan del Corro) sobresale la problemática que presenta la adecuación del proyecto a un solar irregular para sacar el máximo provecho al aforo y del edificio cinematográfico proyectado por Eugenio Aguinaga en una ladera de Bilbao para uso exclusivo para enfermos tuberculosos.

Aparte de estas referencias, hay que destacar que en este mismo año de 1951 aparece un número especial, el 117, dedicado exclusivamente a las salas de cine en España, en donde la información es muy somera pero siempre está acompañada de material gráfico, Figura 4.

Los edificios que se recogen en esta publicación son los siguientes: los cines Consulado (46) (S. Zuazo) y el Olimpia (47) (Luis M. de Gana) en Bilbao; el Pompeya (48) (Juan Pan da Torre), El Rex y el Narváez (49) (Gutiérrez Soto) en Madrid, Figura 5; el Coso (50) (José de Yarza) al que hay que sumar otra sala también de Yarza en colaboración con Ubago en Zaragoza, así como el cinematógrafo Fraga de la ciudad de Vigo proyectado por (51) Gutiérrez Soto. En 1952 se describe un edificio diseñado por (52) Ambrosio Arroyo de uso mixto: hotel más cine, así como el diseñado para cine, viviendas y oficinas en Bilbao de (53) Eugenio M. Aguinaga. En el año 1953 aparece la reforma en dos fases del cine Fémina de Barcelona dirigida por al arquitecto (54) Antonio Moragas Gallissá. Este edificio albergó uno de los primeros cinematógrafos barceloneses y cuenta con dos entradas diferentes para las
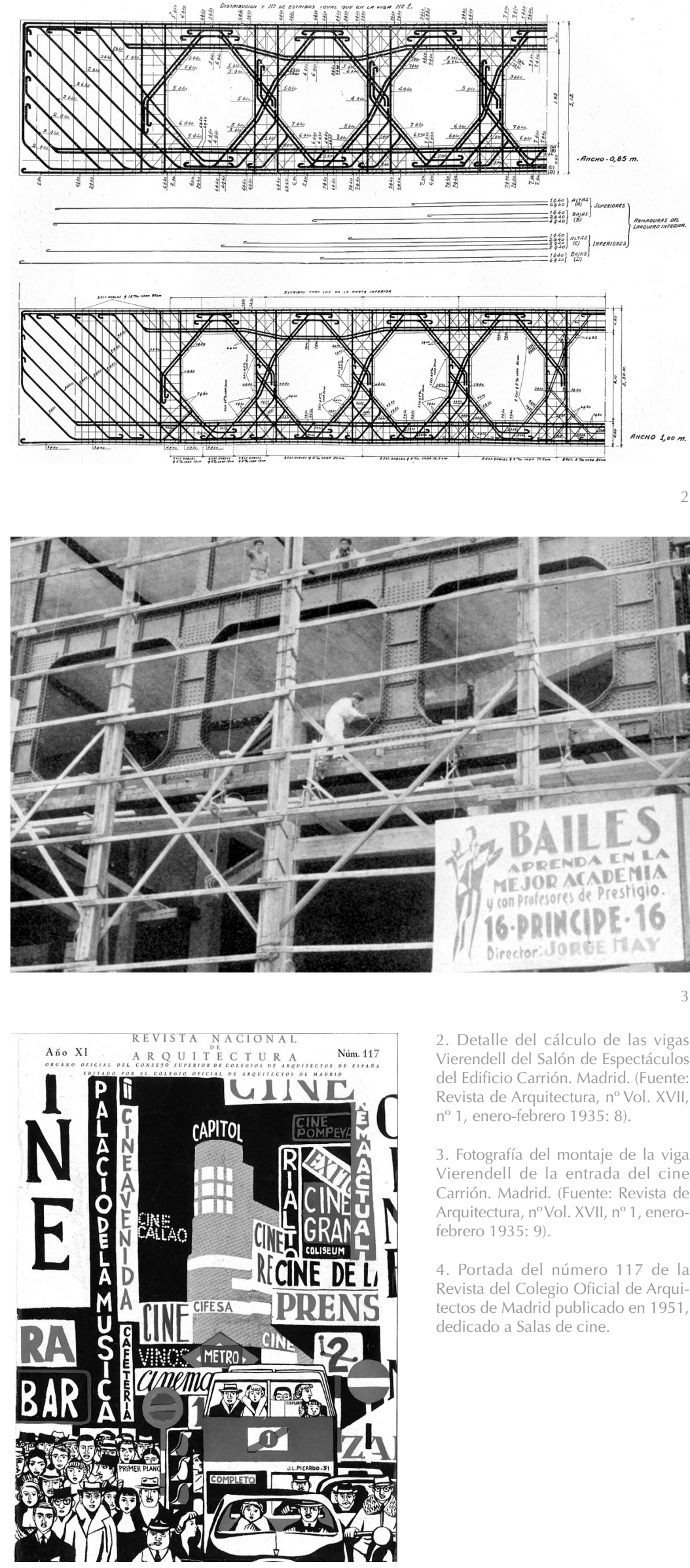

2. Detalle del cálculo de las vigas Vierendell del Salón de Espectáculos del Edificio Carrión. Madrid. (Fuente: Revista de Arquitectura, $\mathrm{n}^{\circ}$ Vol. XVII, no 1 , enero-febrero 1935: 8).

3. Fotografía del montaje de la viga Vierendell de la entrada del cine Carrión. Madrid. (Fuente: Revista de Arquitectura, $\mathrm{n}^{\circ} \mathrm{Vol}$. XVII, $\mathrm{n}^{\circ} 1$, eneroebrero 1935: 9).

- Portada del número 117 de ta Revista del Colegio Oficial de Arquitectos de Madrid publicado en 1951 dedicado a Salas de cine. 


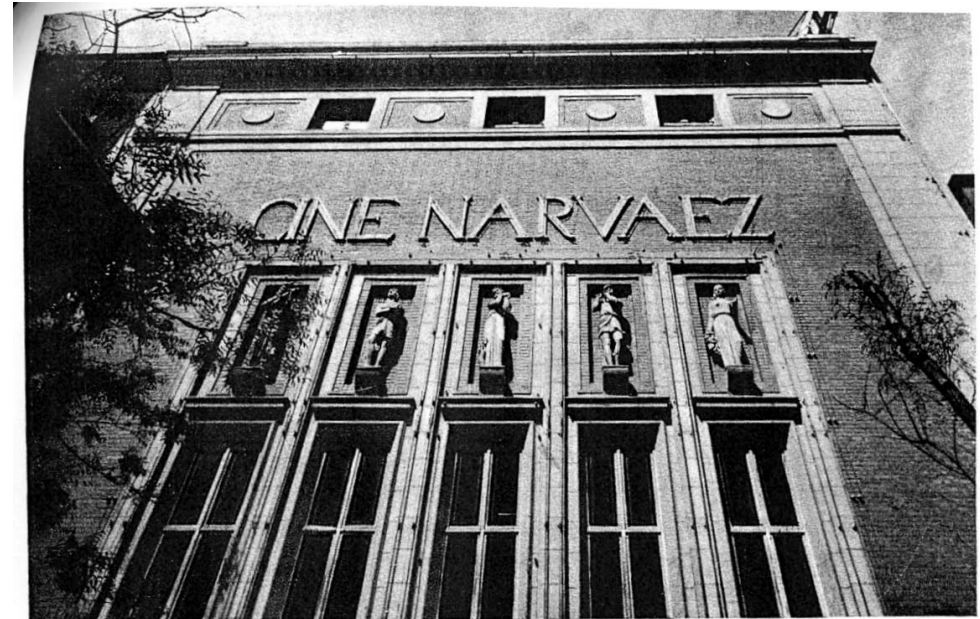

Detalle de la jachadu

\section{G I I N $\quad$ E \\ NARVAEZ}

Luis G. Soto
Arquitecto

Arquitecto

Planta del patio de butacas

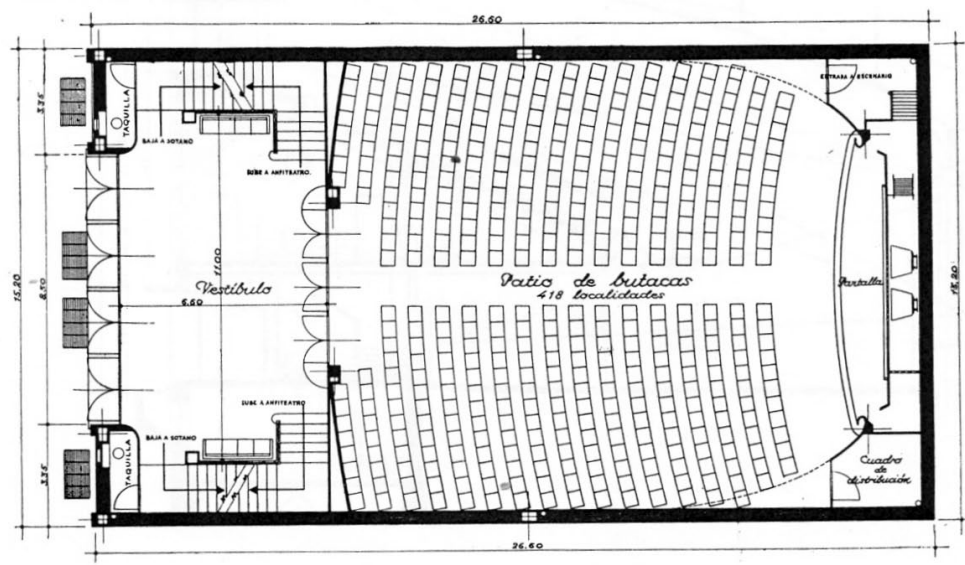

5. Fotografía con parte de la fachada y dibujo de planta del patio de butacas del cine Narváez. Madrid (Fuente: Revista del Colegio Oficial de Arquitectos de Madrid, 117

1951:24).
Cine en Madrid de los llamados de barrio, esto es, para la proyección de películas de reestreno. Con capacidad para 780 localidades entre el patio de butacas y el anfiteatro. Planta construcciones superpuestas o anejas. rectangular en solar con fachada a la calle, sin

forma modesta, a dos cines alemanes de los arquitectos (59) Gascard y Pleiffer.

En los años 60 comienza a descender el número de publicaciones dedicadas a la arquitectura de los cinematógrafos. En esta década sólo aparecen tres artículos. Dos de ellos dedicados a remodelaciones; uno trata el cine Eslava de Valencia (60) (Jiménez de la Iglesia) que se justifica por la necesidad de adaptar el local a los nuevos sistemas de proyección TOD-AO que obliga a modificar el formato de pantalla; el otro versa sobre la conversión de un frontón en sala de cine. El tercer artículo da noticias sobre un edificio de Hellín (61) (Albacete), proyectado en 1966 para viviendas y en cuyos bajos se ubicó una sala de cine. A lo largo de los 70 solamente se hace referencia por medio de dos fotografías al cine Consulado de Bilbao. Además también se dan noticias sobre un edificio mixto que alberga dos salas de cine superpuestas: el cine Goya y el Consulado instalados en los bajos del edificio junto con la cafetería y la discoteca. El texto forma parte de un monográfico sobre la arquitectura burgalesa contemporánea (62). En la década de los 80 con la llegada de las nuevas tecnologías de reproducción fílmica, decae espectacularmente el cine como oferta de ocio y esparcimiento para las masas, la revista deja de dar noticias sobre los edificios cinematográficos ya que prácticamente no se construyen. Solamente aparecen referencias a edificios antiguos como la celebración del 50 aniversario en 1982 del cine Capitol (63) o el homenaje a Anasagasti y sus obras entre las que destaca el Real Cinema proyectado en 1920 (64).

También se cita como noticia la reforma del cine Flix de Tarragona en 1981 (65).

\section{-Informes de la Construcción}

distintas clases sociales. El hecho de que la reforma se llevase a cabo en etapas (19491951) provocó la existencia de dos estilos diferenciados en el mismo edificio. En 1955 se describe de modo sucinto el cine Princesa de Madrid de (55) José Luis Sanz Magallón. En 1959 se dan noticias sobre el cine Marvi de Madrid (56) (Felipe Heredero y Carlos Sobrini) y un cine en Bilbao proyectado por (57) José Luis Sanz Magallón. En estos dos artículos redactados por los autores de los proyectos se describen de forma elemental las características de los edificios a los que acompañan material gráfico. El final de la década se cierra con las noticias sobre la construcción del Motocine de Madrid (58) (Chueca Goitia) y las novedades técnicas que se emplean en la creación de nuevos espacios cinematográficos en los que prima tanto el cálculo y diseño de la pantalla como el planeamiento urbanístico que este tipo de espacio requiere. Además en esta década se vuelven a hacer referencias, de
En 1948 aparece la revista Informes de la Construcción con periodicidad mensual (66), "dedicada a tener al corriente a los técnicos de habla española, sobre las novedades que se producen en el campo de la construcción y sus materiales" (67). Su precursora fue: Anales del Instituto Técnico de la Construcción y la Edificación, editada por el Instituto Técnico de la Construcción y la Edificación entre los años 1934-1946.

En esta publicación encontramos el primer trabajo relativo al cine en uno de sus primeros números (4, 1948: 146-1-146-6). Concretamente, se ofrece una descripción pormenorizada acerca de cómo construir una cabina de proyección, resaltando aspectos técnicos relativos a la ventilación, la protección contra el fuego, distribución, uso de materiales y tipos de suelos, paredes y techos, e incluso tipos de equipos e instalaciones. Todo ello 
acompañado de planimetrías y dibujos de detalles constructivos relevantes, Figura 6.

Algo que está acorde con lo comentado sobre la legislación vigente, en cuanto a que la seguridad, especialmente las medidas contra incendios son los temas más preocupantes y para los que se desarrolla más articulado.

En esta línea de trabajos generales o recomendaciones para construcción de salas de cine, destaca también la presentación sobre el concurso para tres tipos de sala, recogida en el número 7 de la revista de 1948 (Techniques et Architecture: 3-4). J. Kittrich hace un repaso a cómo cambian las salas de cine desde su nacimiento hasta 1948 (68), destacando que la acústica y la técnica de iluminación son determinantes tanto en la forma y en la decoración no sólo de interiores, sino también del exterior de los edificios (69), Figura 7. Es decir, se resalta el carácter funcionalista de esta arquitectura del cine, adaptada a las necesidades del espectáculo.

Igualmente, y como una de las aportaciones más novedosas de cómo debe ser el cine, en especial desde el nacimiento de la televisión y como arte que compite frente a ésta, están los trabajos y comentarios del famoso arquitecto cineasta (70) Ben Schlanger. En 1949, en el número 14 de la revista, bajo la denominación de "Nueva arquitectura del cine" (71) se recogen una serie de comentarios de este autor sobre cómo deben realizarse los cines. Sus sugerencias más originales van encaminadas a la supresión del marco que encuadra la imagen, aumento y movilidad de la pantalla y mejora en la naturalidad del ambiente mediante una iluminación de la sala en relación constante con la luminosidad de la pantalla. La acústica es otro de los temas que preocupa, especialmente para que el sonido enfatice la calidad dramática de la obra que se expone. Junto con todo ello resalta la importancia de la sencillez de la decoración, confort del local y aspecto social del cine en la colectividad humana. En su opinión, el arquitecto debe tener muy en cuenta la función social del cine. Estos comentarios se complementan con tres ejemplos de obras suyas. Sea como fuere, a excepción de estas tres valoraciones generales sobre cómo construir salas de cine, el resto de aportaciones a la revista sobre cines son de carácter individual y ejemplarizantes. Desde 1948 hasta 1978, puesto que más adelante no se ha hecho, se publican un total de 22 salas de cine, incluidas las tres mejores presentadas al concurso referido anteriormente. De ellas, 20 son publicadas con anterioridad a la década de los 60 y sólo dos más, una en 1967 ( n$^{\circ} 195$, cine Victoria en Hospitalet de Llobregat) y otra en 1978

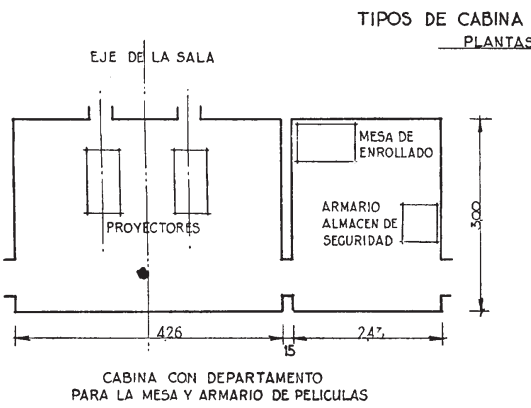

(1)
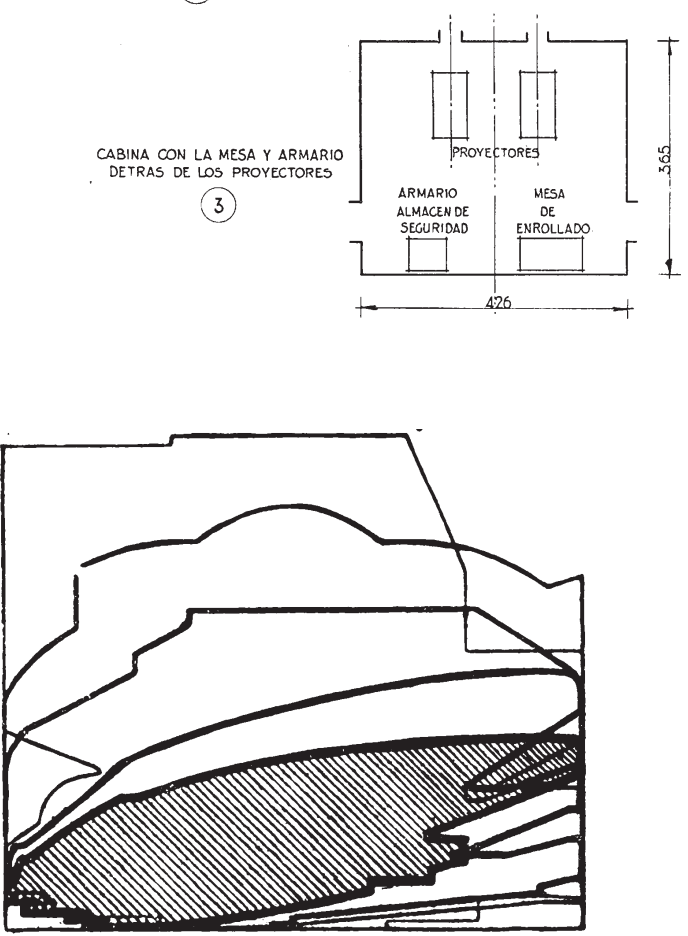

Fig. 1.-Influencia de la visibilidad en las salas de espectáculos (ejemplos de 1850 a 1930, dibujados a la vez más bajios y butacas cada vez más elevadas.

$\left(n^{\circ} 304\right.$, teatro-cine Eileen Norris, California EEUU) lo hacen con posterioridad a estas fechas. Ello es muestra de que son los años 40 , y especialmente los 50, en los que hay un claro interés por este tipo de construcciones y que confirman lo observado en el resto de revistas valoradas.

La diferencia es que frente a los cuatro trabajos recogidos en Arquitectura o Revista Nacional de Arquitectura y uno recogido por Cortijos y Rascacielos, en Informes de la Construcción, el peso de los trabajos extranjeros y en consecuencia, la búsqueda de novedades técnico constructivas más allá de nuestras fronteras está claramente acentuada. A excepción de 4 artículos (72), todos son salas construidas en otros países y por arquitectos de fuera de nuestras fronteras. Concretamente, se trata de cines americanos y europeos. Destacan cuatro números de la revista que recogen más de una sala por cada uno. Ya comentamos uno de ellos, los tres
6. Tipos de cabinas de proyección. (Fuente Informes de la Construcción n%. 1948: 146-1).

7. Influencia de la visibilidad en las salas de espectáculos. (Fuente: Informes de la Construcción nº 7. 1948: 146-2). 
8. Imagen del interior del cine Studio Theater de Manhattan. (Fuente: Informes de la Construcción n 14. 1949: 146-4). proyectos del concurso de tipos fundamentales de salas de cine recogidos en el número 7 de la publicación, donde la acústica y la iluminación tienen un peso muy significativo a la hora de abordar las formas del edificio proyectado, algo que entonces era pionero y se vinculaba principalmente a la entrada del cine sonoro.

Quizás de mayor interés y que anteriormente anunciábamos, puesto que sus obras se realizan en distintos países y demuestran la fama y especialidad en cinematógrafos que adquiere este arquitecto, son las tres salas recogidas en el número 14 de la revista (1949). Nos estamos refiriendo a trabajos realizados por el norteamericano Shlanger, en dos ocasiones en codirección con Hoffberg, Reisner y Urbahn (73), que sin duda, han sido base e inspiradores para otros. Seguramente seleccionados por sus singularidades, se recoge una sala en Bermuda, donde se llama la atención sobre la construcción de una pantalla flotante.

Un estudio teatro en Manhattan (Nueva York), Figura 8, donde el juego con la iluminación es determinante para la sala y, por último, un cine en Lima en donde según se comenta, se encontraba el cine con pisos más planos del mundo, compatible con una buena visión de la pantalla.

Ya en 1951, en el número 34 de la revista, volvemos a encontrar cuatro ejemplos significativos de cines italianos y franceses, en lo que podemos considerar un especial de salas de espectáculos. Por sus singularidades, destacan el realizado en Nápoles (cinemateatro Metropolitan), donde su arquitecta (74) (Filo-Speziali), necesitó un apoyo importante de Ingeniería puesto que la sala fue excavada en roca. De la parte francesa, más por su reconocimiento internacional que por el detalle de la información ofrecida sobre el cine, destaca la construcción del Palacio de los Festivales Internacionales de Cannes, obra de (75) Maurice Gridane.

Por último, es el número 60 de Informes de la Construcción el que recoge más salas de cine, entre ellas las tres españolas comentadas con anterioridad, más una suiza y otra en (76) Hannover. Quizás, lo que más destaca en este número es un texto a modo de reflexión acerca de cómo hacer salas de espectáculo, en donde la funcionalidad, determinada por la visibilidad y acústica, cobra un protagonismo acorde con lo que se observa en los distintos proyectos de la época, señalando que la misma no impide un buen desarrollo estético, siendo el proyectista el que debe combinar adecuadamente estas tres facetas de la construcción de este tipo de edificios.

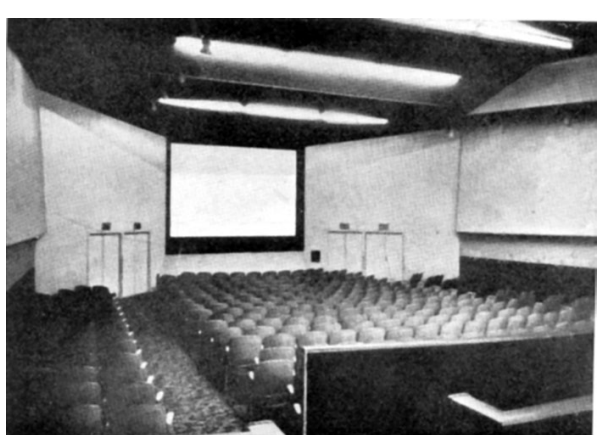

8

En el resto de números de esta revista en los que hay salas de cine, se recogen obras sueltas, no por ello menos significativas que las señaladas, y con la característica de que casi siempre, la sala aparece combinada con otro tipo de actividades o servicios para la edificación. Por ejemplo, el caso del cinema (77) Hotel de Sao Paulo o el centro de radio y cinema de Cuba).

En cuanto al contenido de todas estas publicaciones sobre salas concretas, vienen a confirmar la lectura hecha por Kittrich y también por Shlanger, en cuanto a la importancia de la función del edificio, destacando otras adaptaciones que resultan necesarias, que van desde los aforos, pasando por los sistemas de ventilación, hasta el confort o la funcionalidad múltiple del edificio en la que se ubica la sala.

\section{CONCLUSIONES}

Del análisis del contenido de las revistas técnicas españolas sobre las salas de cine se ha llegado a las siguientes conclusiones

- La construcción de las salas de cine se encuentra condicionada por el discurso histórico del momento. De este modo puede apreciarse durante el primer tercio del siglo $X X$, un acomodo al eclecticismo arquitectónico imperante y que más adelante dará paso a una arquitectura propia con claras influencias internacionales, especialmente alemana y americana.

- Las propias características de cada una de las revistas objeto de este estudio, (Arquitectura/Revista Nacional de Arquitectura, Cortijos y Rascacielos e Informes de la Construcción) condicionan tanto la información como su presentación. Ello se aprecia como mínimo en los siguientes puntos:

- En Arquitectura/Revista Nacional de Arquitectura la mayoría de salas referenciadas son madrileñas. En cambio, los cines difundidos por Cortijos y Rascacielos recogen información sobre todo el terri- 
torio nacional, mientras que Informes de la Construcción se dirige hacia la publicación de espacios extranjeros como ejemplo de modernidad y nuevas tendencias arquitectónicas.

- Respecto a los autores de los artículos, en Arquitectura/Revista Nacional de Arquitectura suelen coincidir con el responsable del proyecto arquitectónico, mientras que en las otras dos revistas, los trabajos son reseñados principalmente por los editores de las publicaciones.

- En cuanto a la cantidad de textos sobre salas, desde una perspectiva temporal, se puede decir que:

- En la década de los años 30, cuando se inicia el auge del cine como espectáculo, aparece una gran proliferación de artículos sobre este tipo de salas como queda patente en el caso de Arquitectura (las otras dos publicaciones analizadas no existen en esta época).

-Desde los años 30 a los 50 aparecen el mayor número de publicaciones dedicadas a los cinematógrafos y es coincidente con el apogeo del espectáculo como fenómeno de masas del momento histórico.

-A partir de los años 50 comienza el declive de este espectáculo debido, fundamentalmente, a la aparición de la televisión. Este hecho se refleja en el descenso de nuevas salas de cine $y$, por ende, en su desaparición en las publicaciones técnicas.

- Como se ha mencionado, la mayoría de textos se refieren a salas madrileñas pero no se tiene la certeza de que ello se deba únicamente a que sea el Colegio de Arquitectos de Madrid quien edite esta publicación. Probablemente también tenga cierta influencia el hecho de que son en las grandes ciudades donde, por motivos meramente demográficos, se concentren la mayoría de salas dando lugar a una mayor variedad de construcciones.

- Las salas de cine que aparecen entre las páginas de las revistas suelen ser de notables arquitectos bien nacionales o internacionales, que aportaron a la arquitectura del momento importantes innovaciones tanto desde el punto de vista del diseño como de las técnicas empleadas. Los arquitectos españoles más destacados, suelen coincidir con los más renovadores del momento, como por ejemplo, Martínez Feduchi, Eced, Gutiérrez Soto, López Delgado o Zuazo.
- El contenido de los artículos es muy similar en las tres publicaciones, destacando aspectos técnicos como la acústica, el confort, la climatización, la iluminación y la seguridad, siendo su referencia una constante en la legislación técnica. En general, en los artículos prima la economía y optimización de recursos.

- En la mayoría de los trabajos se destacan los materiales utilizados, especialmente interiores, para mejorar la acústica o asegurar las condiciones frente a incendios.

- Es llamativo que los concursos que se recogen no se refieren a salas con gran aforo, sino más bien a cines pequeños (200-300 localidades), para su ubicación en localidades menores o que requieran determinadas adaptaciones climáticas. En cambio, la mayoría de cines que recogen estas revistas son para más de 700 personas. Las complejidades técnicas suelen venir de la mano de la adaptación de los espacios, puesto que rara vez son edificios exclusivamente dedicados al cine, sino que comparten usos con viviendas, locales comerciales e incluso hoteles u otras salas de espectáculo.

- En cuanto a las carencias, se echa en falta un mayor número de publicaciones referidas a espacios al aire libre, que son realmente excepcionales. No obstante, también se nos habla de la precariedad, carácter desmontable y el escaso valor arquitectónico de las salas de este tipo, normalmente utilizadas estacionalmente (los famosos cines de verano) y que hoy, muchas, también han desaparecido.

Como conclusión general, puede decirse que es la funcionalidad y la importancia que se le da al público, lo que marcan las pautas de este tipo de obras, comentándose cómo varios autores, tanto españoles como extranjeros se refieren a ellas. Esta es una razón más para entender el peso socio económico que tuvo el cine en determinado momento, marcando parte de nuestra Historia. De esta etapa, nos quedan películas, guiones, recuerdos fotográficos de actores y actrices y, cómo no, las salas... El valor patrimonial de este tipo de obras y la aportación que suponen las revistas técnicas como fuente documental sobre ellas las convierte, como se anunció, en un referente ineludible para su conservación. Se espera que este trabajo colabore en el mantenimiento de las salas de cine en España y sirva como referente para otros estudios paralelos o de casos concretos sobre este tipo de edificaciones.

\section{AGRADECIMIENTOS}

Este trabajo está enmarcado en el proyecto de I +D HUM2007-65543/HIST. 


\section{REFERENCIAS Y NOTAS}

(1) De la Madrid, J.C. (coord.) Primeros tiempos del cinematógrafo en España. TREA. Oviedo (1996b) pp.348.

(2) La prensa se sigue haciendo eco de esta destrucción, como por ejemplo la reciente desaparición del Cine Goya en Granada (El www.ideal.es, 23/06/08). Otro ejemplo significativo es que desde 2004, momento en que el Ayuntamiento de Madrid cambió su Plan General, desprotegiendo el uso "dotacional" de los cines, 14 salas han cerrado en las calles más céntricas de la capital (Vera Gutiérrez y Daniel Verdú (007) Reportaje "El cine Avenida también se rinde. En www.elpais.com, 01/02/2007).

(3) Se trata del Cine Coliseo en Eibar (Vizcaya, País Vasco). BOE 21/07/1987 (incoación) y 19/08/1988 (desestimación de la incoación).

(4) Seguin, J.C. y Letamendi, J. "El sistema Lumière en España (1896-1897" en De la Madrid, J.C. (coor.) 1996b: 25-49.

(5) González López, P. "Pioneros y creadores de cine mudo catalán. 1896-1914" en De la Madrid J.C. (coor.) 1996: 217-237.

(6) Pérez Rojas, F.J. "Los cines madrileños del barracón al rascacielos" en VVAA 1986: tomo II.

(7) De la Iglesia Salgado, F., Moreno Pérez, J.R., Pérez Humanes, M. y Ruíz Robles, A.: Arquitectura teatral y cinematográfica. Andalucía 1800-1990. Junta de Andalucía. Consejería de Cultura y Medio Ambiente. Sevilla (1990), pp. 319.

(8) Carrasco García, M.: "Los inicios de la Arquitectura cinematográfica en Soria" Celtiberia, Vol. 42 $\mathrm{n}^{\circ} 84$ (1992), pp. 371-388.

(9) De la Madrid, J.C. Cinematógrafo y "varietés" en Asturias. 1896-1915. Consejería de Cultura. Principado de Asturias (1996a), pp. 413.

(10) Fernández Muñoz, A.L. Arquitectura Teatral en Madrid. Del corral de comedias al cinematógrafo. El Lavapiés. Madrid (1989), pp. 472.

(11) Ibid.: Fernández Muñoz, pp. 473-278.

(12) Juan Antonio Ramírez. La Arquitectura en el Cine. Hollywood, La Edad de Oro. Ed. Hermann Blume. 1986.

(13) Aunque hubo pruebas previas, se considera que fue en 1956 cuando comienza su emisión la primera televisión en España (Televisión Española: http://www.rtve.es/).

(14) Balsalobre García, J.M. Arquitectura de salones cinematográficos y cine. Universidad de Alicante. (2006), pp. 133.

(15) Ibid: De la Iglesia Salgado, et al. (1990).

(16) Biel Ibañez, M.P.: "El cine como documento para la historia de la arquitectura industrial en Zaragoza: Antonio de Pauda Tramullas" Artigrama, Vol.11 (1994-95), pp. 297-315.

(17) Aunque de forma menos focalizada que en este trabajo, los tratados de construcción - Neufer, Ernst: Arte de Proyectar en Arquitectura. Consultor para arquitectos, ingenieros, aparejadores, estudiantes, constructores y propietarios. Ed. Gustavo Gili, S.A. 1961. Cinematógrafos. pp. 338-343-, las biografías de los grandes arquitectos especialistas en el diseño de cinematógrafos - Baldellou, Miguel Ángel: Luis Gutiérrez Soto. Colección Artistas Españoles Contemporáneos, Dirección General de Bellas Artes, Ministerio de Educación y Ciencia. Madrid 1973. Carlos de Miguel (Coordinador): La obra de Luis Gutiérrez Soto. COAM. 1978. Luís Gutiérrez Soto. Catálogo de la Exposición organizada por la Dirección General de la Vivienda, La arquitectura y el Urbanismo del Ministerio de Fomento. Sociedad Editorial Electa España, S.A. Madrid 1997; Las Salas de Cine. pp. 300-330 - o las guías de arquitectura como la del Colegio de Arquitectos de Madrid -Guía de Arquitectura y Urbanismo de Madrid. Tomo I y II. COAM. 1982 y Arquitectura de Madrid. Fundación COAM, Madrid. 2003aportan también una valiosa información sobre localización, proyectistas, fechas y a veces algunos datos más de sumo interés para el estudio y la evolución de estos espacios.

(18) A la hora de valorar estos aspectos y seleccionar las fuentes de análisis de este estudio se han tenido en cuenta los siguientes trabajos: García Melero, José Enrique; Azorín López, V. y Sorli Rojo, A. "Los repertorios bibliográficos españoles sobre las artes plásticas". Art Libraries Journal, 14 (3) 1989, Azorín López, V. y Sorli Rojo, A.: "Las bases de datos con información artística" Revista de Documentación Científica, 16 (2) 1993 y Sorli Rojo, A., Fernández Caballero, D., Mochón Bezares, G., y Azorín López, V. "Las Publicaciones periódicas de construcción en España y su importancia en la transferencia de conocimientos" Actas de las II Jornadas de Investigación en Construcción. 2008. Instituto de Ciencias de la Construcción. CSIC. Madrid.

(19) Concretamente, son trabajos referidos a salas de multicines o modernas del tipo de los IMAX (Image Maximum, Máxima imagen creado por IMAX Corporation que tiene la capacidad de proyectar representaciones de mayor tamaño y definición que los sistemas aleatorios de proyección), que incluso a veces, van ligados a la construcción de planetarios u otros usos: Calatrava, S. "El Ojo Cósmico. Cine-planetario en la Ciudad de las Artes, Valencia" Arquitectura Viva, n 61. Jul-Ago. 1998: 34-36. Correa Suárez, L. "Rehabilitación del cine Cuyás y su entorno. Las Palmas de Gran Canaria" Basa, n²3. 2000: 96-104 (Se trata de una rehabilitación para conversión del cine en auditorio moderno y centro cultural).Garcés, J. y Sòria, E. "Cine Imax en el Port Vell, Barcelona. AV. Arquitectura y Vivienda. Monografías 57-58, Ene-Abr. 1996.: 114-117.Kugel, K. "Iceberg y Bunker. Coop Himmelbau, un palacio de cine en Dresde" Arquitectura Viva, nº 62. Sep.-Oct. 1998: 52-56. 
(20) Las normas sobre salas de cine que principalmente afectan a los años analizados en este texto son las siguientes:

1. Capítulos del XII al XVII del Reglamento de Policía de Espectáculos, de construcción, reforma y condiciones de los locales destinados a los mismos. Real Orden del Ministerio de la Gobernación, de 19 de octubre de 1913. Gaceta de Madrid, n³04. 31/10/1913: 347-355.

2. Real Orden Circular, del Ministerio de la Gobernación, de 26 de Febrero de 1922. Coello. Gaceta de Madrid, n 61. 02/04/1922:928, que hace especial hincapié en la prohibición de cinematógrafos en cafés y restaurantes, costumbre muy extendida, sin que cumplan lo articulado para la seguridad del público, recogido en el Reglamento de 1913.

3. Orden, del Ministerio de la Gobernación, de 15 de agosto de 1933. Casares Quiroga. Gaceta de Madrid, $n^{\circ}$ 234: 1221, por la que se modifica el reglamento, concretamente en relación a la entrada del sonido, en relación a la seguridad, y se expone textualmente: “...el verdadero peligro de incendio que con el cinematógrafo mudo sólo radicaba en las cabinas, previsto y resuelto en el Reglamento de Espectáculos, ha sido trasladado, además, a los escenarios u hornacinas con el advenimiento del cinematógrafo sonoro, lo que obliga a establecer condiciones especiales de seguridad de la instalación del mismo...",

4. Capítulo III de la Primera Parte y artículos 156 al 166 de la tercera parte y capítulos XVI y XVII del Reglamento de Policía de Espectáculos públicos. Orden, del Ministerio de la Gobernación, de 8 de mayo de 1935. Gaceta de Madrid, no 125, 05/05/1935:1055-1070. Este último es sustituido por el de 1982, que ha estado vigente hasta la promulgación del código técnico de la edificación.

(21) Debe advertirse que aunque se citan algunas por su importancia, como puede ser el Palacio de la Música de Madrid, en este estudio se han evitado las salas de espectáculo, puesto que muchas de ellas el cine no tiene un papel tan significativo y apenas se resaltan sus detalles constructivos en estas publicaciones.

(22) S. Ulargui: "Cine Actualidades" Cortijos Y Rascacielos, № 14 (1933) , pp. 28-32

(23) Aníbal Alvarez: "Proyecto de cine al aire libre" Cortijos Y Rascacielos, № 16 (1934), pp. 21-24

(24) José González Edo: “Un cinematógrafo en Málaga" Cortijos Y Rascacielos, No 24, (1944), p. 24

(25) Felipe López Delgado: "Cine "Coruña" en La Coruña" Cortijos Y Rascacielos, № 25 (1944), p. 14

(26) Pedro Ricardt Biot: "Cine "Montecarlo", en Barcelona" Cortijos Y Rascacielos, NNo 31 (1945), p. 4

(27) Santiago Lagunas Mayandía: "Cinematógrafo en Borja (Zaragoza)", Cortijos Y Rascacielos, № 41 (1947), p. 19

(28) Eduardo Robles: "Cine Avenida, en San Luis de Potosi" Cortijos Y Rascacielos,, NNo 50 (1948), p. 28

(29) Cine Vallehermoso de Manuel López Mora (1945, n² 27), el cine de la calle Marqués de Urquijo $\left(1945, n^{\circ} 30\right)$ y el cine con viviendas de la calle Luchana (1946, n' 37) ambos de Alemany y Muñoz Monasterio, así como el cine Margerit de Villaverde Alto de José Luís Durán de Cottes (1947, n 40)

(30) Zuazo, S. : "Cine Consulado en Bilbao", Revista Arquitectura №117 (1951), pp. 1-4.

(31) Gutiérrez Soto, L.: Revista Arquitectura "El cine Callao" № 14 (1927), pp. 57-66.

(32) Lozano Lardet, E. : Revista Arquitectura "Cine de San Carlos" Agosto (1929), pp. 304-309.

(33) García Mercadal, F.: Revista Arquitectura "La última obra de Poelzig" № 14 (1926), pp. 353-358.

(34) Mendelsohn, E.: Revista Arquitectura "El cine "Universum" en Berlín" Febrero (1929), pp. 67-69.

(35) "Fallo del Concurso Nacional de Arquitectura, 1931" Revista Arquitectura Febrero (1932) pp. 48-55.

(36) López Delgado, F.:“Teatro Fígaro (Madrid)” Revista Arquitectura Febrero (1932), pp.56-61.

(37) Riancho y Torriente. "Cine Tetuán" Febrero Revista Arquitectura (1932), pp.62- 65.

(38) Martínez Feduchi, L. y Eced V. :"El edificio Carrión" Revista Arquitectura (1935), pp. 3-21.

(39) Sanz de Bergue, J. y Fonseca Llamedo, J.:"El cine Carretas" Revista Arquitectura (1935), pp. 246-266.

(40) Alonso Martos, F.:"El cine Salamanca" Revista Arquitectura (1936) pp. 91-97.

(41) López Izquierdo, E. y Durán de Cottes, J.L.: "El Cinema-Palace" Revista Nacional de Arquitectura (1944), pp.127-131.

(42) Aguinaga, E.M. y Domínguez Salazar, J.A. "Cine Novedades de San Sebastián" Revista Nacional de Arquitectura (1946), pp. 61-66.

(43) Lagunas, S.: "Reforma del Cine Dorado" Revista Arquitectura (1949), pp. 510-512

(44) Prieto Moreno, F. :"Cine "Aliatar", en Granada" Revista Arquitectura (1947), pp. 143-149.

(45) Aixelá J.M. y Tárrega, M.A. :“Cine Niza en Barcelona" Revista Arquitectura (1951), pp. 32.

(46) Zuazo Ugalde, S. :"Cine Consulado en Bilbao" Revista Arquitectura (1951), pp. 62.

(47) De Gana, L.M.:"Cine Olimpia en Bilbao" Revista Arquitectura (1951), pp. 18-20.

(48) Pan da Torre, J.: "Cine Pompeya" Revista Arquitectura (1951), pp. 5-7.

(49) Gutiérrez Soto, L.: "Cine Rex en Madrid" Revista Arquitectura (1951), pp. 21-23 y "Cine Narvaez" №117 (1951) pp. 24-25.

(50) De Yarza, J.: - "Cine Coso" №117 Revista Arquitectura (1951), pp. 8-10.

(51) Gutiérrez Soto, L.: "Cine Fraga en Vigo" Revista Arquitectura (1951), pp. 14-17.

(52) Arroyo, A. : "Hotel, con Cine, en Segovia" Revista Arquitectura (1952), pp. 18-22.

(53) Eugenio M. Aguinaga "Edificio para cine, viviendas y oficinas en Bilbao" Revista Arquitectura (1952), pp.16-25

(54) De Moragas Gallissá, A.: "Cine Fémina, en Barcelona" Revista Arquitectura (1953), pp. 31-34

(55) Sanz Magallón, J.L.: -"Cine Princesa, en Madrid" Revista Arquitectura (1955), pp. 45-50.

(56) Heredero, F. y Sobrini, C. : "Cine Marvi" Revista Arquitectura (1959), pp. 31-34. 
(57) Sanz Magallón, J.L.: - "Cine en Bilbao" Revista Arquitectura (1959), pp. 33-36.

(58) Chueca Goitia, F. y Bello Lasierra, S.: "Motocine" Revista Arquitectura (1959), pp. 29-32.

(59) Gascard, D. :"Cine Thega en Hildesheim" Revista Arquitectura (1951), pp. 40.

(60) Jiménez de Laiglesia, L.: "Cine Eslava en Valencia" Revista Arquitectura (1962), pp. 31-33.

(61) Garaulet y Pérez Blanco: "Cine y viviendas en Hellín" Revista Arquitectura (1966), pp. 23.

(62) De Abajo Ontañón, F. y Gutiérrez Ruíz, P.: "Edificio Cines "Goya" y "Consulado" Burgos. Año 1961" Revista Arquitectura (1974), pp. 71-73.

(63) "Antología. El edificio Capitol en el cincuentenario de su construcción (Matinez Feduchi y Eced" Revista Arquitectura (1982), pp. 57-67.

(64) "Real Cinema" y "Cine Madrid-París" (Homenaje Anasagasti)", Revista Arquitectura (1983) pp. 16-18 y pp. 40-43.

65) Fernández, F., Gallego, M. y Pasarius, H.: "Reforma del cine-teatro Flix" Revista Arquitectura (1983): 54.

(66) Aunque tiene carácter mensual, solamente se editaban en la primera época 11 números anuales. En julio-agosto solía aparece un solo ejemplar.

(67) Informes de la Construcción. Vol. II, n 12, 1949. p.2. IETCC-CSIC.

(68) F.L. :"Cabina de proyección de cine Informes de la Construcción, Vol. 4: 146-1(1948) pp. 1-6.

(69) Kittrich, J.:"Salas de cine. Concurso de proyectos sobre el tema. "Tres tipos fundamentales". Informes de la Construcción, № 3-4 (1948): "Technique et Architecture".

(70) Schlanger, B.: - "Un cine con iluminación modulada" Informes de la Construcción, №14: "Architectural record" 146-4 (1949) pp. 5-6.

(71) Pons Sorolla, F. :"Nueva arquitectura del cine" Informes de la Construcción, № 2. no 14: s.p.

(72) Concretamente, se trata del trabajo ya citado del Cine Victoria (Hospitalet), obra de Barba Corsini. Los otros tres se recogen en el número 60 del año 1954. Uno es un cine rural, en Berja, provincia de Almería, cuya autoría es de Joya y Rebolledo (146-38). Y los otros dos pertenecen al conocido arquitecto Gutiérrez Soto. Se trata de los cines Bulevar y Amaya. Ambos en Madrid, en el caso del segundo en colaboración con De Aguinaga (146-39).

(73) Hoffberg, Reisner y Urbahn :"Cine con "pantalla flotante" en Bermuda". Informes de la Construcción, No 14: "Architectural record" 146-4 (1949a) pp. 7-12.

(74) Galzenati, M.: "Cinema-Teatro Metropolitan, en Nápoles. Il cemento. Arquitecto: Stefania Filo Speziali. Ingenieros: M. Aprato, F. Penta y A. Galli" Informes de la Construcción, No 34: 146-16 (1951), pp.1-4.

(75) "Palacio de los festivales Internacionales en Cannes. L'Architecture francaise. Arquitectos: Maurice Gridaine" Informes de la Construcción, No 34: 146-12 (1951b), pp.1-3.

(76) "Cine Aegi en Hannover. Bauwelt. Arquitectos: H. Kluppelberg y G. Lichtenhahn", Informes de la Construcción, Nº 60 (1954c): 146-34.

(77) J.M.A.:-"Cinema-Hotel en Sao Paulo. Arquitecto: Rino Levi" Informes de la Construcción, Vol. 16 (1949) "Centro de radio y cinema, en Cuba. Arquitectos: Jungo, Gastón y Domínguez" Informes de la Construcción, Nº 20 (1950): "L'Architecture d'Aujourd'hui". 